\title{
15 colour photometry of the Landolt SA95 standard star field $\star, \star \star$
}

\author{
Xu Zhou ${ }^{1}$, Zhaoji Jiang ${ }^{1}$, Jun Ma ${ }^{1}$, Suijian Xue ${ }^{1}$, Hong $\mathrm{Wu}^{1}$, Jiansheng Chen ${ }^{1}$, Jin Zhu ${ }^{1}$, \\ Weihsin Sun $^{2}$, and R. A. Windhorst ${ }^{3}$
}

\author{
1 National Astronomical Observatories, Chinese Academy of Sciences, Beijing 100012, PR China \\ 2 Institute of Astronomy, National Central University, Chung-Li 32054, Taiwan \\ 3 Department of Physics and Astronomy, Box 871504, Arizona State University, Tempe, AZ 85287-1504, USA
}

Received 23 April 2002 / Accepted 18 September 2002

\begin{abstract}
In this paper, we present a set of photometric observations in 15 colors of stars in the Landolt SA95 field with the BATC system. The wavelengths covered by the system range from $300 \mathrm{~nm}$ to $1000 \mathrm{~nm}$. Visual magnitudes of the stars studied in the field are from the 10th to 20th mag. The observational methodology and the data reduction procedures are described. The relationships between the BATC intermediate-band system and the Landolt $U B V R I$ broad band system are obtained. A catalogue of the photometry has been produced which contains the SEDs of 3613 stars.
\end{abstract}

Key words. methods: observational - techniques: photometric - stars: general

\section{Introduction}

Landolt (1983) gave his measurements of $U B V R I$ magnitudes of 233 standard stars in a strip about two degrees wide centered on the celestial equator for the purpose of homogeneous flux calibration from both hemispheres. Landolt (1992) presented the $U B V R I$ photometry of another 298 standard stars around the celestial equator, within a visual magnitude range of 11.5-16.0 and a $B-V$ colour range from -0.3 to +2.3 . The Landolt catalogues have been widely used by observers using intermediate to large size telescopes. By using the Landolt standard catalogues, Galadí-Enríquez et al. (2000) obtained $U B V R I$ photometry of 11 different fields around the Landolt standards and gave 681 secondary standards with a visual magnitude range from 9.7 to 19.4 and a $B-V$ range from 1.15 to 1.97.

With the capability of imaging large fields, BeijingArizona-Taipei-Connecticut (BATC) survey program has observed a number of selected fields which include the Landolt SA95 field centred at $\alpha=3 \mathrm{~h} 54 \mathrm{~m} 17 \mathrm{~s}, \delta=+00^{\circ} 19^{\prime} 08^{\prime \prime}(2000.0)$. So far the Landolt SA95 has been one of the fields with highest observing quality. Thus, we select it to demonstrate the photometric quality of the BATC system. Standard BATC data reduction processes have been applied. In this field, 7 stars have been measured in Landolt (1983), and 45 are listed in the

Send offprint requests to: $\mathrm{Xu} \mathrm{ZHOU}$,

e-mail: zhouxu@bac.pku.edu.cn

* The work is partly supported by Chinese National Sciences Foundation under the contract No. 19833020 and No. 19503003.

$\star \star$ The catalogue is only available at the CDS via anonymous ftp to cdsarc.u-strasbg.fr (130.79.128.5) or via

http://cdsweb.u-strasbg.fr/cgi-bin/qcat?]/A+A/397/361 catalogue of Landolt (1992). In addition, 7 stars were included in the secondary standard catalogue of Galadí-Enríquez et al. (2000).

The main purpose of this paper is to present the spectral energy distributions (SEDs) of the field stars in the format of 15 colours, and to show the relationships between the BATC and the $U B V R I$ photometric systems derived by using the stars in the catalogues of Landolt (1983), Landolt (1992), and Galadí-Enríquez et al. (2000). In Sect. 2, we present the observing processes of the Landolt SA95 field. In Sect. 3, we describe the method of data reduction. The magnitude error of observation and data reduction are discussed in Sect. 4. The discussion of the system transformation is given in Sect. 5. Section 6 gives the conclusions of this study.

\section{Observation}

Large field multi-colour photometry has been and is still being conducted with the BATC photometric system. The major observing facility employed is the $60 / 90 \mathrm{~cm} \mathrm{f} / 3 \mathrm{Schmidt}$ Telescope located at Xinglong Station of Beijing Astronomical Observatory (BAO). A Ford Aerospace $2048 \times 2048$ CCD camera with a 15 micron pixel size is mounted at the Schmidt focus of the telescope. The field of view of the CCD is $58^{\prime} \times 58^{\prime}$ with a plate scale of 1.7 arcsec per pixel. An image of the Landolt SA95 field taken with this system in the $i$ band $(666 \mathrm{~nm})$ is shown in Fig. 1.

The BATC filter system includes 15 intermediate band filters, covering a range in optical wavelengths from 300 to 1000 nm (Fan et al. 1996; Yan et al. 1999; Zhou et al. 2001). The filters are designed specifically to avoid contamination 


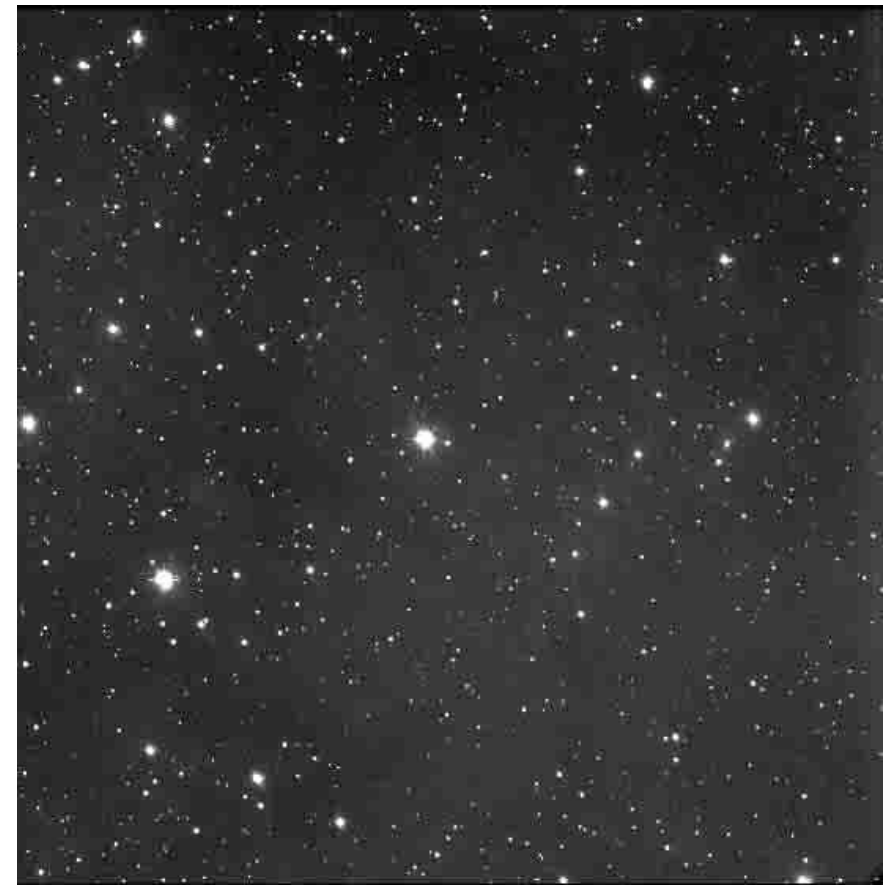

Fig. 1. An $i$ filter image of the Landolt SA95 field with a field of view of $58^{\prime} \times 58^{\prime}$.

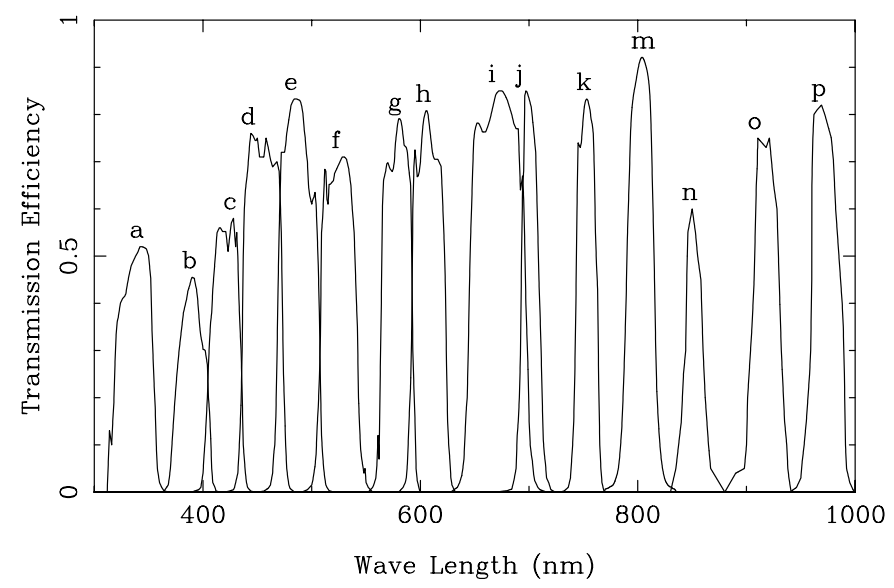

Fig. 2. The transmission curves of the 15 BATC filters. The filter codes (see Table 1) are labelled on top of each curve.

from the brightest and most variable night sky emission lines. The transmission curves of the 15 filters are given in Fig. 2.

The BATC photometric system defines the magnitude zero points in a way similar to the spectrophotometric $\mathrm{AB}$ magnitude system. The AB system is a monochromatic $\widetilde{f}_{v}$ system first introduced by Oke \& Gunn (1983), based upon the spectral energy distributions (SEDs) of the four $F$ sub-dwarfs, HD 19445 , HD 84937, BD+262606, and BD+17 4708.

A great advantage of the $\mathrm{AB}$ magnitude system is that the magnitude is directly related to physical units. As in the definition of AB magnitude system, the BATC magnitude system is defined as:

$m_{\text {batc }}=-2.5 \cdot \log \widetilde{F_{v}}-48.60$,

where $\widetilde{F_{v}}$ is the flux per unit frequency in unit of $\operatorname{erg~s}^{-1} \mathrm{~cm}^{-2} \mathrm{~Hz}^{-1}$.

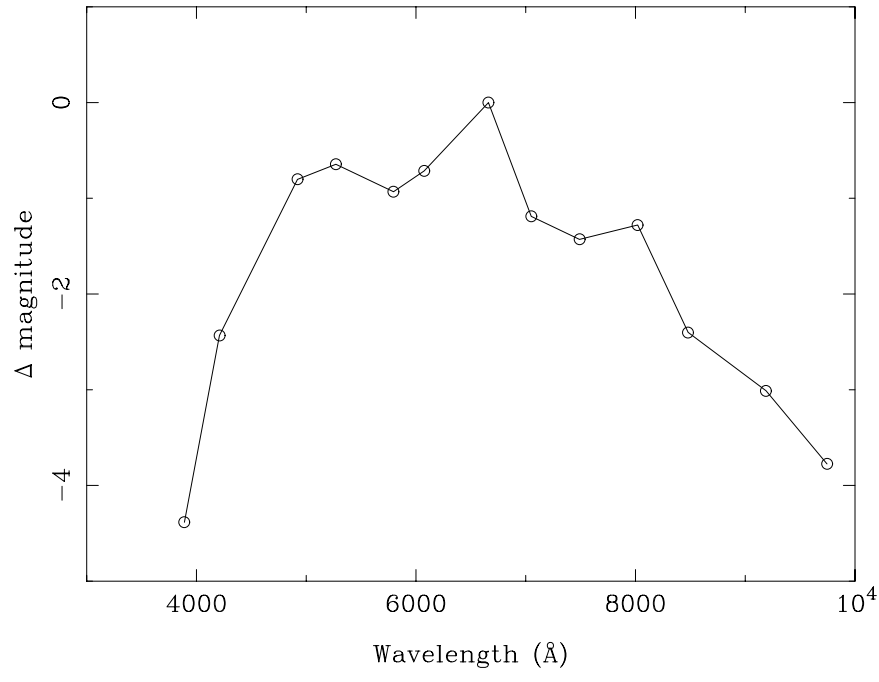

Fig. 3. The spectral sensitivity curve of the BATC photometric system obtained from the observations of standard star HD 84937 in 13 bands. The magnitude difference is normalized to $i$ band $(666 \mathrm{~nm})$.

In the BATC system (Yan et al. 1999), $\widetilde{F_{v}}$ is defined as

$\widetilde{F_{v}}=\frac{\int \mathrm{d}(\log v) f_{v} R_{v}}{\int \mathrm{d}(\log v) R_{v}}$,

which links the magnitude to the number of photons detected by the CCD rather than to the input flux (Fukugita et al. 1996). The response of the system, $R_{\lambda}$, which is used to relate $f_{v}$ and $\widetilde{F_{v}}$, includes only the filter transmissions. Other effects such as the quantum efficiency of the CCD, the response of the telescope's optics, and the transmission of atmosphere, etc., are ignored. This makes the BATC system filter-defined, since the bandwidths are intermediate in size and all the responses are essentially flat within each specified passband.

The magnitudes of the 4 Oke \& Gunn (1983) standards have subsequently been refined by many authors (Oke 1990; Castelli \& Kurucz 1994), with Fukugita et al. (1996) presenting the latest re-calibrated fluxes of these four standards. Their magnitudes in the BATC system have been slightly corrected recently using the data obtained on a number of photometric nights (Zhou et al. 2001).

In order to understand the sensitivity curve of the BATC photometric system, we ran a test observation of HD 84937 on a night of good observing quality (Zhou et al. 1999). With equal exposure time, we obtained the images in 13 BATC filter bands. The differences between instrumental magnitude and BATC magnitudes of this star can be regarded as the sepectral sensitivity of the BATC photometric system. The result is shown in Fig. 3. The magnitude is normalized to the $i(666 \mathrm{~nm})$ band. The spectral sensitivity curve includes all effects of the system such as the CCD response, filter band width and its transmissions, and the transparency and reflection efficiency of telescope optics, and the spectral extinction of the atmosphere. From this figure, we can see immediately that the $i(666 \mathrm{~nm})$ band is the most sensitive one in the BATC photometric system, while the $b(389 \mathrm{~nm})$ and $p(975 \mathrm{~nm})$ bands are about 4 mag less sensitive. 
Table 1. Statistics of observations.

\begin{tabular}{ccccccc}
\hline \hline No. & Filt & $\begin{array}{c}\lambda \lambda \\
(\mathrm{nm})\end{array}$ & $\begin{array}{c}\text { Exp. } \\
\text { (hour) }\end{array}$ & $\begin{array}{c}\text { Num. of } \\
\text { images }\end{array}$ & $\begin{array}{c}\text { Num. of } \\
\text { Calib. }\end{array}$ & $\begin{array}{c}\text { Calib. } \\
\text { error }\end{array}$ \\
\hline 1 & $a$ & 337.15 & $2: 17$ & 6 & 2 & 0.001 \\
2 & $b$ & 390.69 & 5.50 & 34 & 4 & 0.033 \\
3 & $c$ & 419.35 & 0.96 & 7 & 2 & 0.000 \\
4 & $d$ & 454.00 & 1.33 & 9 & 4 & 0.007 \\
5 & $e$ & 492.50 & 2.30 & 8 & 2 & 0.010 \\
6 & $f$ & 526.68 & 1.22 & 6 & 7 & 0.007 \\
7 & $g$ & 578.99 & 2.23 & 10 & 8 & 0.007 \\
8 & $h$ & 607.39 & 1.33 & 8 & 7 & 0.007 \\
9 & $i$ & 665.59 & 1.75 & 11 & 11 & 0.006 \\
10 & $j$ & 705.74 & 4.22 & 16 & 9 & 0.004 \\
11 & $k$ & 754.63 & 3.30 & 13 & 6 & 0.008 \\
12 & $m$ & 802.32 & 3.69 & 17 & 6 & 0.006 \\
13 & $n$ & 848.43 & 3.73 & 12 & 7 & 0.004 \\
14 & $o$ & 918.22 & 4.40 & 14 & 3 & 0.006 \\
15 & $p$ & 973.85 & 4.15 & 15 & 3 & 0.006 \\
\hline
\end{tabular}

We have observed the Landolt SA95 field on a total of 41 nights in the period from December 13, 1994 to December 16, 1999. A total of 189 high-quality images were selected for further photometric analysis. The total exposure time and image number of each filter band are listed in Table 1. A typical long exposure time lasts for 10 to $20 \mathrm{~min}$, while it is $5 \mathrm{~min}$ for short exposure. The short exposure images were taken during photometric nights for flux calibration.

In the nights judged photometric by the observers, the standard stars of Oke \& Gunn (1983) were observed between air-masses 1.0 and 2.0 for each programmed filter band. The standards were observed with short exposures to avoid saturation. Normally, there is enough time to obtain flux calibration images for 4 to 6 filters on each photometric night. In each image, the standard star is located at or near the center.

A subsection of the $300 \times 300$ pixels on CCD was used in these standard star observations in order to save readout time and disk space. The extinction coefficients and magnitude zero points obtained from the standard star observations are then used to calibrate other BATC field images. The observations for calibration are described in detail in Zhou et al. (2001).

\section{Data reduction}

\subsection{Pipeline I}

An automatic data reduction procedure called "Pipeline I" has been developed as a standard for the BATC multi-colour sky survey (Fan et al. 1996), which includes bias subtraction and flat-fielding of the CCD images. The dome flat-fielded images in each filter were subsequently combined. When combining the images, the cosmic ray hits and bad pixels were corrected through a comparison of multiple images. The reduction and combination of Landolt SA95 images was proformed in the same way.

Before the combination, the images were shifted and rotated according to the precise locations of stars in the HST
Guide Star Catalogue (GSC) (Jenkner et al. 1990). A plate solution of 8 parameters were obtained by positioning the GSC stars of the images in each colour. The positions of the stars in the images $(x, y)$ then can be easily transformed to the equatorial coordinates ( $\alpha$ and $\delta$ ). The final RMS errors in positional accuracy of the stars are about 0.5 arcsec.

\subsection{Pipeline II}

A "Pipeline II" program has been developed to measure the magnitudes of point sources in BATC images. It is based on Stetson's standard procedure of DAOPHOT (Stetson 1987). The Pipeline II includes 4 major steps:

1. to find all the sources in the images;

2. to perform aperture photometry at 10 different radii of apertures;

3. to construct PSF parameters and a look-up table with bright isolated stars, and

4. to perform PSF fitting to each point source and obtain its magnitude.

In the first step, objects are identified in the combined images. An object detected at least in 3 bands is considered real. Certain objects that appear at the edge of some images are marked in the final output SED catalogue.

In a series of tests with different image profiles, we find that the analytical profile "moffat 25 " best fits the observed PSF in the BATC images. In a one degree field, the FWHM of the PSF can change 0.2 arcsec from one place to another. This is due to the fact that the Schmidt focal plane is curved while the CCD surface is flat (Fan et al. 1996).

To obtain a quasi-linear variation for the PSF lookup table, we divided each image into $3 \times 3$ sections with some overlap between adjacent sections. In each such section, we normally select more than 20 bright, isolated stars for PSF fitting with the "moffat25" profile, and obtain a look-up table to trace the linear residual of the PSFs across the sub-images.

In our automatic procedures of data reduction, most of the parameters in each step were optimised and fixed, leaving only a few, such as the $F W H M$ of the object, to vary according to the seeing conditions at the time the image was taken.

It is difficult to obtain good PSF fitting for faint stars due to their asymmetry in profile. However, in order to construct an SED catalogue as complete as possible, faint stars were measured with aperture photometry using an aperture with a diameter of 9 pixels. The difference between PSF fitting and aperture photometry is corrected using the bright, isolated stars in the same field. These faint stars are marked in the catalogue.

At the completion of photometry, the SEDs of all the measurable objects are obtained. All of these objects have also been visually inspected. There are usually some special objects, such as small extended sources, unresolved blended stars, and ghost images around very bright stars, etc. These special objects are marked, along with the objects close to saturated stars. 


\subsection{Calibration}

Using the images of the standard stars observed on the photometric nights, we derive iteratively the extinction curves and the slight variation of the extinction coefficients with time (Zhou et al. 2001). The extinction coefficients at any given time in a night $[K+\Delta K(U T)]$ and the zero point of the instrumental magnitude $(C)$ were obtained.

The instrumental magnitudes $\left(M_{\text {inst }}\right)$ of selected bright, isolated stars can be readily transformed to the BATC AB magnitude system $\left(M_{\text {batc }}\right)$ by

$M_{\mathrm{batc}}=M_{\mathrm{inst}}+[K+\Delta K(U T)] \cdot \chi+C$

where $\chi$ is the airmass at which the image was taken, and $C$ is the zero point of instrumental magnitude.

These bright stars, for which the calibrated magnitudes were obtained in short exposures, were taken as secondary standards and were subsequently used to tranform the magnitudes in the process of calibration. Normally about 30 stars, which are brightest, isolated, and unsaturated, were selected to perform calibration on the combined image of each filter.

The error in magnitude for a single star is about $0.03 \mathrm{mag}$, a value obtained from the comparison of the magnitudes in a short exposure with that from the combined image. The mean error propagated using 30 stars should thus be less than $0.01 \mathrm{mag}$.

The flux calibration for the images taken on the 18 photometric nights has been done several times.

We then take the mean value of calibration and obtain a final calibration error for each filter. Table 1 lists the times that calibration has been performed and the calibration error for each filter. The error of calibration, Error, listed in the last column of Table 1 was calculated using

Error $=\sqrt{\frac{\sum(\mathrm{mag}-\overline{\mathrm{mag}})^{2}}{n(n-1)}}$.

In most filters, the calibration errors are equal to or less than $0.01 \mathrm{mag}$. The errors in $a$ and $b$ bands (337 and $390 \mathrm{~nm}$ ) are about $0.02 \mathrm{mag}$, larger than that in the other bands. This may come from the fact that the seeing effect is worse in shorter wavelengths, and the bright stars are not as bright as in other bands due to the lower intensity of the stars as well as the lower sensitivity of the CCD in shorter wavelengths.

A naive guess will predict that the error in the $a$ band should be larger than that in $b$, but the opposite was observed. This may be due to small number statistics. The same effect may also present in the errors of certain bands with a small number of calibrations. In general, except for $a$ and $b$ bands, the total calibration error is about $0.01 \mathrm{mag}$.

The final SED catalogue of the Landolt SA95 field is created also in electronic format. In this catalogue, each star takes up 2 lines. The first line contains the coordinates and magnitudes of the stars in 15 bands. The second line gives the estimated error of the magnitudes. For the stars also found in the USNO all-sky catalogue, their USNO names and $B$ and $R$ magnitudes are presented at the beginning of the second line. There are a total of 3613 stars in the catalogue, listed in order of increasing right ascension.

The catalogue of photometry is available in electronic form at the Centre de Données astronomiques de Strasbourg (CDS) via anonymous ftp to cdsarc.u-strasbg. $\mathrm{fr}$ (130.79.128.5).

\section{The total measurement error}

\subsection{The sources of magnitude error}

The total error of calibration comes from the following sources: a) bias (over-scan) correction and CCD readout noise $(0.001 \mathrm{mag})$; b) flat-field correction $(0.003 \mathrm{mag})$; c) photometry of PSF fitting (0.02 mag); d) calibration error (0.01 mag).

We employed the Lick Observatory data-collection system, which automatically subtracts the overscan of each image during readout, and records the overscan in the last column. We then process each program image through median filtering the original overscan and adding back to the image the difference between the original subtracted overscan and this filtered overscan. In this way, the signal-to-noise ratio $(S / N)$ of the overscan is increased and any residual large-scale pattern produced in the image by imperfections in the overscan is removed. The overscan will increase the noise value of the image background. The magnitude error arising from the bias depends on the brightness of the star, and it can be treated as the background error in photometry.

Normally, 12 dome flat-field images in each band were taken within 24 hours before and/or after the observations. To avoid shutter effects, the exposure time is always longer than 2 min.

The number of ADU of each flat-field image is higher than 20000 . We estimate the error of the flat-field correction to be $0.3 \%$ (Fan et al. 1996; Zhou et al. 2002). When the statistical error is considered, the flat-field error in the Landolt SA95 field is about 0.003 mag.

By comparison with different flux calibrations, the calibration error is estimated to be about $0.01 \mathrm{mag}$. Thus the main error in the magnitude in the catalogue is the photometric error of PSF fitting. It comes from the statistical photon counting error and the sky background noise. The value of these photometric measurement errors is dependent on the brightness of the objects. In our catalogue, a magnitude-dependent error is given for each object. For brighter stars, the errors are about $0.02 \mathrm{mag}$. For fainter stars, the magnitude errors are relatively larger, depending on their magnitudes.

\subsection{Test of total measurement error}

In order to test the error estimate of our photometry, we made an observation of the Landolt SA95 field in $i$ band $(666 \mathrm{~nm})$. As shown in Fig. 4, the test images were taken at positions along the top edge aligned with the top two corners of the Landolt SA95 field. Through the same data reduction processing, we obtained the magnitudes of the objects in the test images. The photometric error is obtained by comparing the magnitudes of the objects in the overlapping area of the test and the combined 


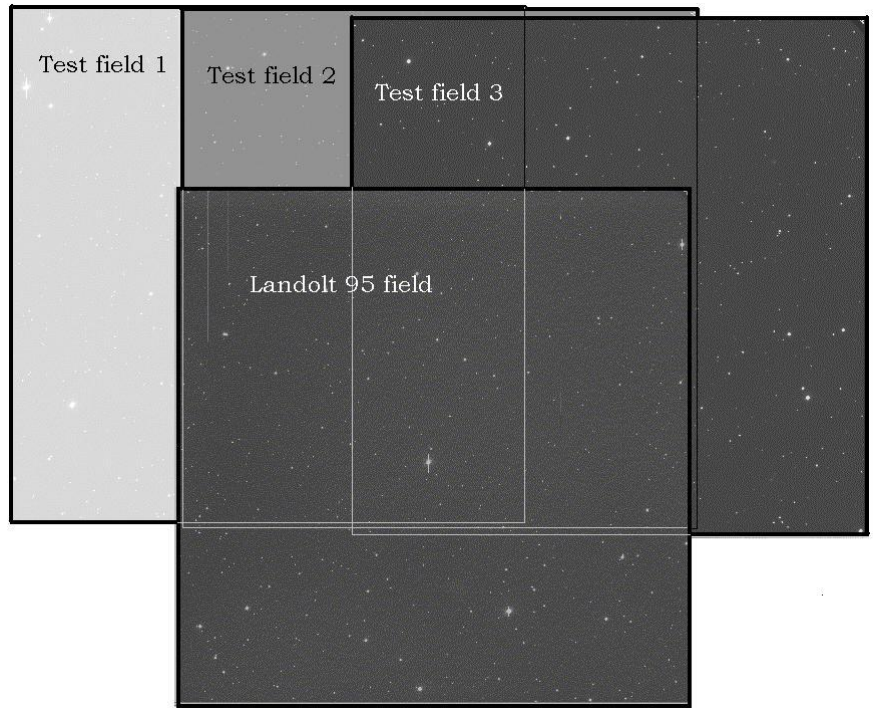

Fig. 4. The positions of the BATC Landolt SA95 field and the test fields.

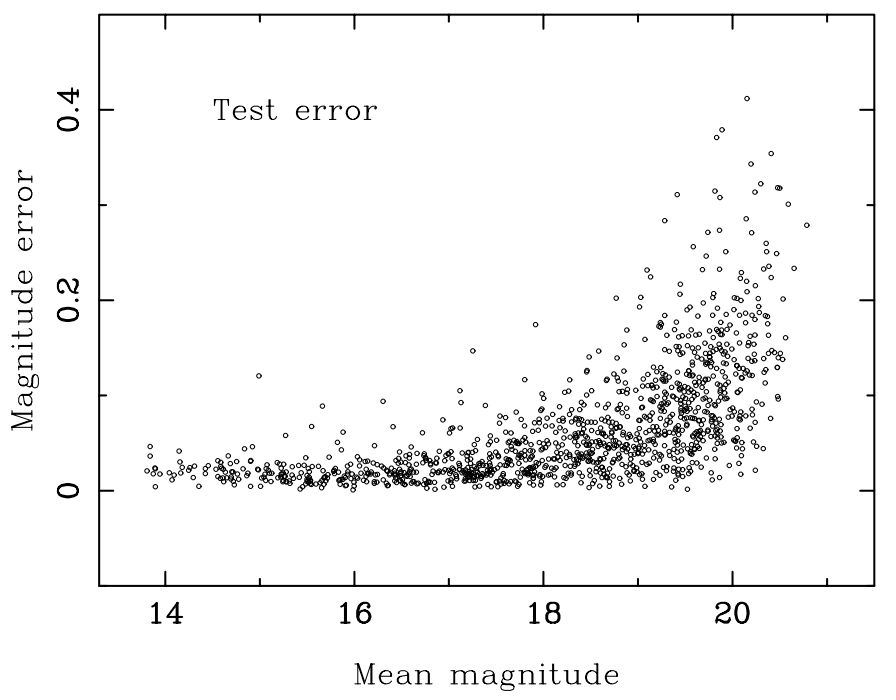

Fig. 5. The diagram of magnitude error versus magnitude in $i$ band $(666 \mathrm{~nm})$. The error is the magnitude differences between the magnitude obtained from the test images and the combined image of SA95 field.

Landolt SA95 images. Figures 5 and 6 show this comparison. The circles in Fig. 5 represent the real magnitude difference between the test image and the Landolt SA95 image, and the plus signs in Fig. 6 represent the magnitude errors given in the catalogue of the test image. From these two figures, it is apparent that the errors in the catalogue show the same distribution as the real measurement errors.

\subsection{Magnitude limit and completeness of the catalogue}

Two concepts have been adopted to define the quality of the measurements in the resulting catalogue. The first one is the limiting magnitude of the observation, and the other one is the completeness of the catalogue. The limiting magnitude is

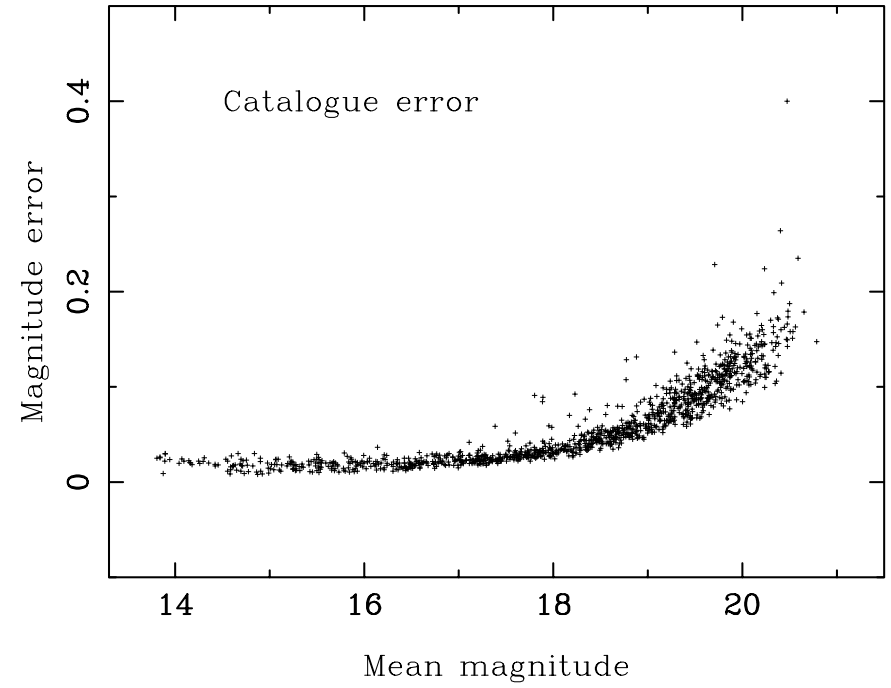

Fig. 6. The diagram of magnitude error versus magnitude in $i$ band $(666 \mathrm{~nm})$. The magnitude error is the measurement error listed in the catalogue.

Table 2. Magnitude limit $(\Delta \mathrm{mag}=0.1)$ and completeness defined by the maxima in the magnitude histogram.

\begin{tabular}{ccc}
\hline \hline filter & limit of mag. & completeness of mag. \\
\hline$a$ & 18.9 & 20.5 \\
$b$ & 19.6 & 21.0 \\
$c$ & 19.1 & 20.5 \\
$d$ & 19.8 & 21.0 \\
$e$ & 20.5 & 22.0 \\
$f$ & 20.2 & 21.0 \\
$g$ & 20.2 & 20.5 \\
$h$ & 19.9 & 21.0 \\
$i$ & 20.3 & 20.5 \\
$j$ & 20.3 & 20.0 \\
$k$ & 19.8 & 19.5 \\
$m$ & 19.0 & 19.5 \\
$n$ & 18.6 & 19.0 \\
$o$ & 18.6 & 19.0 \\
$p$ & 17.6 & 18.5 \\
\hline
\end{tabular}

defined as the magnitude at which the mean magnitude error of the star becomes $0.1 \mathrm{mag}$. By fitting the distribution of the error bars along the magnitudes, we obtained the curve of the error bars as a function of the magnitude,

$\Delta \operatorname{mag}=a_{1} 10^{(\mathrm{mag} / 2.5)}+a_{0}$,

where $a_{0}$ and $a_{1}$ are constants obtained in the fitting process.

From the curve we obtain the limiting magnitude for each band and the error of $0.1 \mathrm{mag}$. The completeness of the catalogue, which is also expressed as a magnitude, is defined as the maximum of the star counts as a function of the magnitude. It shows how many stars can be detected brighter than a certain magnitude limit, which gives an estimate of the completeness of the sample. We have plotted the histogram of magnitudes for each filter band with a binning width of $0.5 \mathrm{mag}$. 


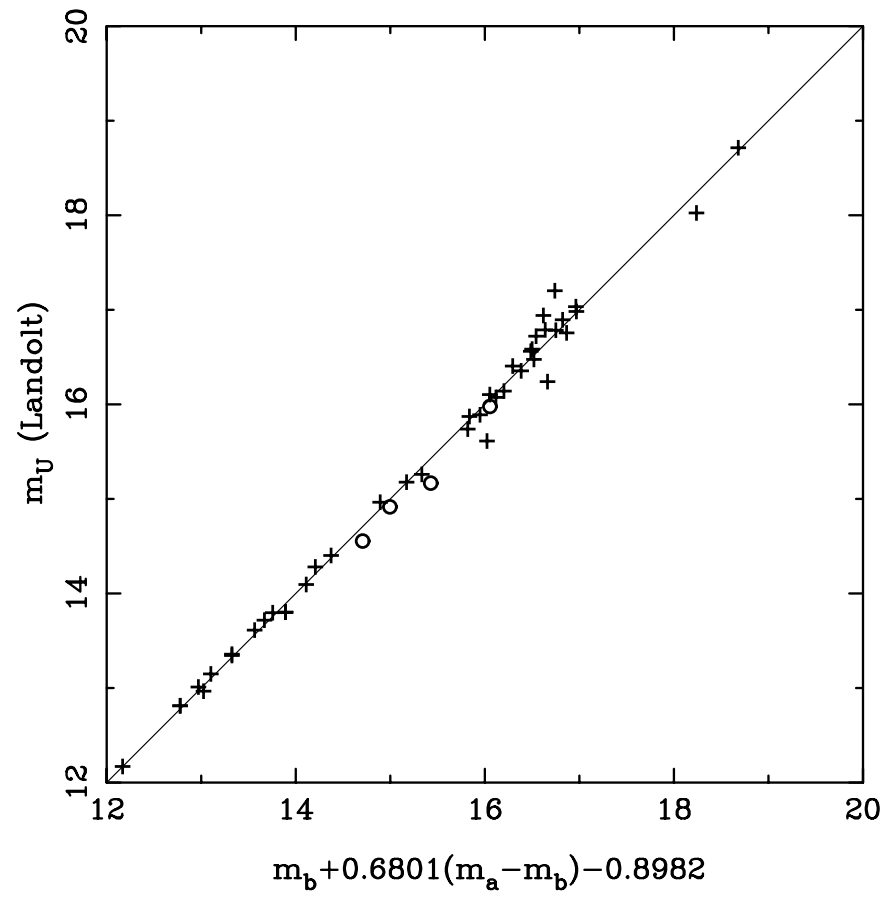

Fig. 7. The comparison between Landolt $U$ magnitude and the BATC $a$ and $b$ magnitudes. Here $b(391 \mathrm{~nm})$ band is the filter band nearest to $U$ band, and $a(337 \mathrm{~nm})$ and $b(391 \mathrm{~nm})$ bands are used for colour correction.

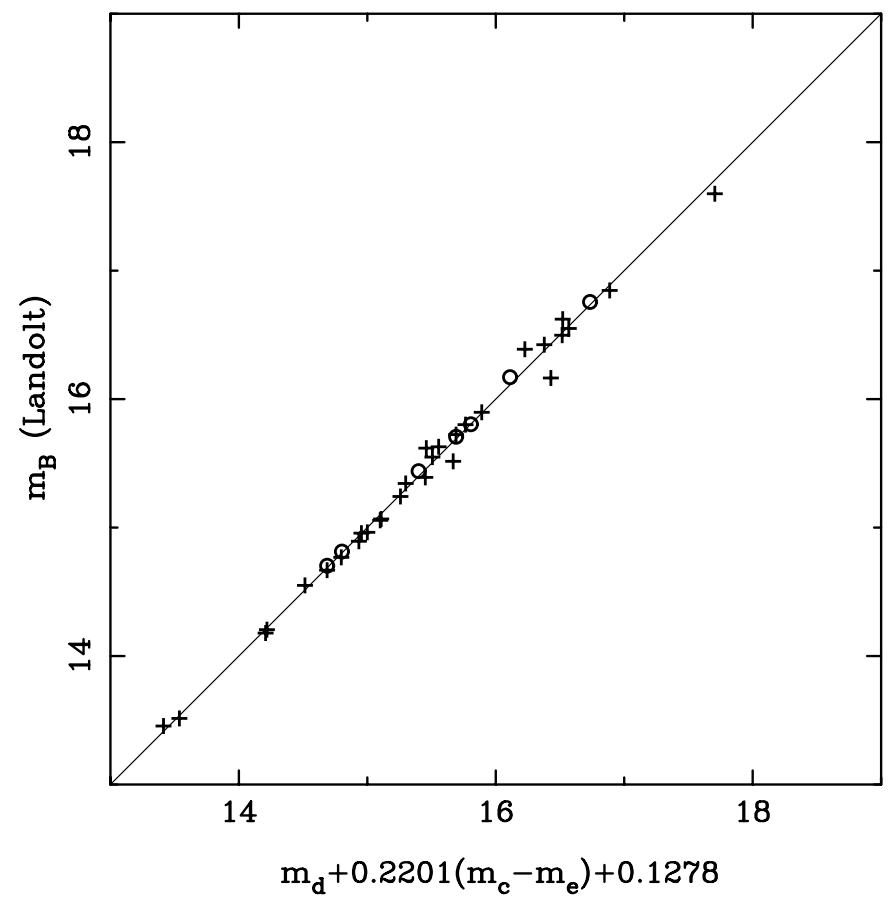

Fig. 8. The comparison between Landolt $B$ magnitude and the BATC $d, c$ and $e$ magnitudes. Here $d$ band (454 nm) is the filter band nearest to $B$ band, and $c(419 \mathrm{~nm})$ and $h(493 \mathrm{~nm})$ bands are used for colour correction.

The maximum of each magnitude bin is taken to be the indicator of the completeness.

The results of limiting magnitude and the estimated completeness are shown in Table 2. In this table, we can see that the limiting magnitudes of the $a$ and $p$ filter bands are about 2 mag

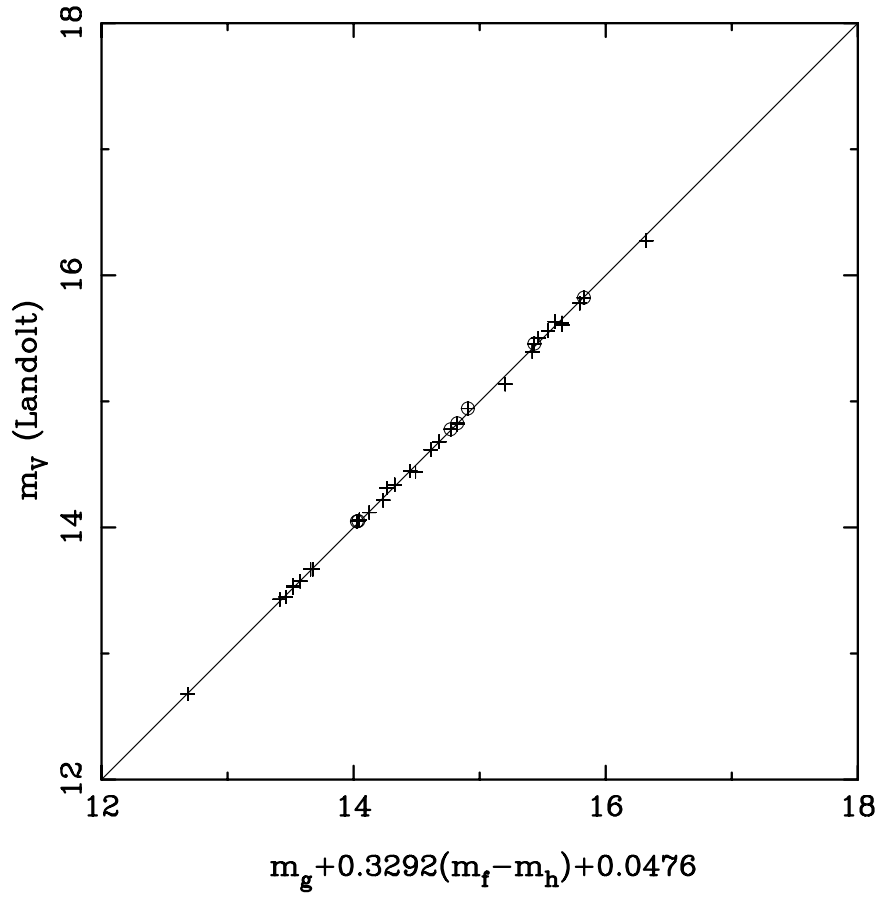

Fig. 9. The comparison between Landolt $V$ magnitude and the BATC $g, f$ and $h$ magnitudes. Here $g$ bands $(580 \mathrm{~nm})$ is the filter band nearest to $V$ band, and $g(580 \mathrm{~nm})$ and $h(607 \mathrm{~nm})$ bands are used for colour correction.

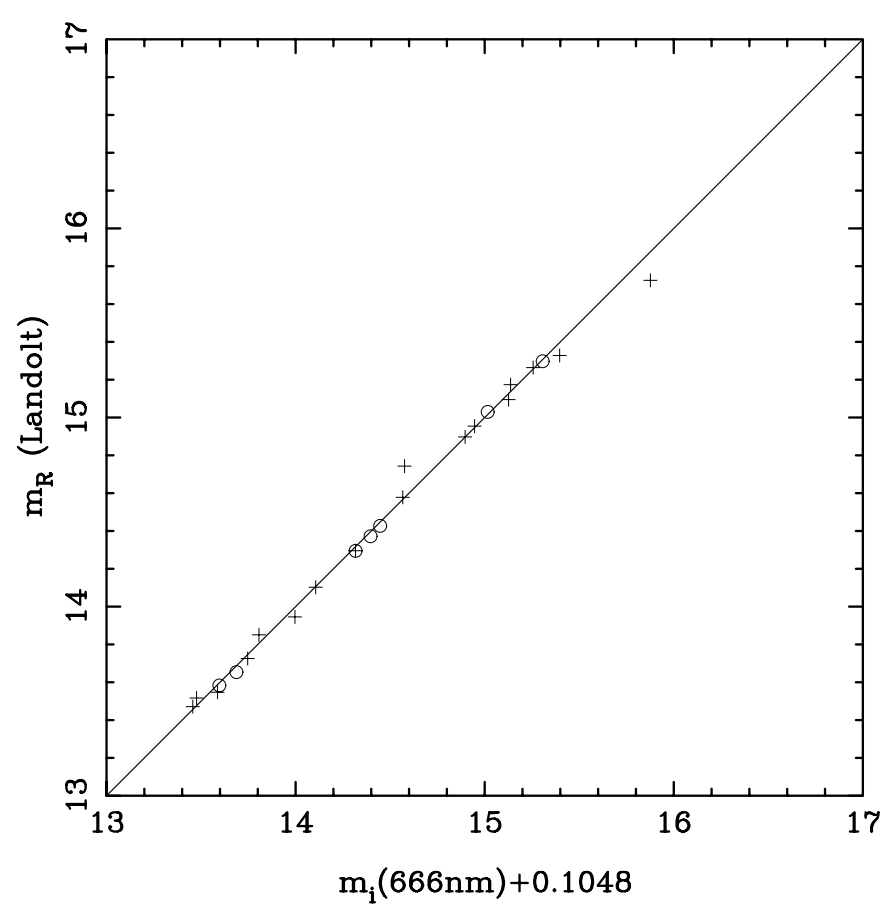

Fig. 10. The comparison between Landolt $R$ magnitude and the BATC $i(666 \mathrm{~nm})$ band. Colour correction does not improve the quality of the fit in this case.

brighter than the $i$ band, even though the exposure time in these two bands is much longer than that in the $i$ band. Another interesting point from the table is that the difference in the completeness among various bands is less than that of the limiting magnitude. The reason is that aperture photometry was performed 


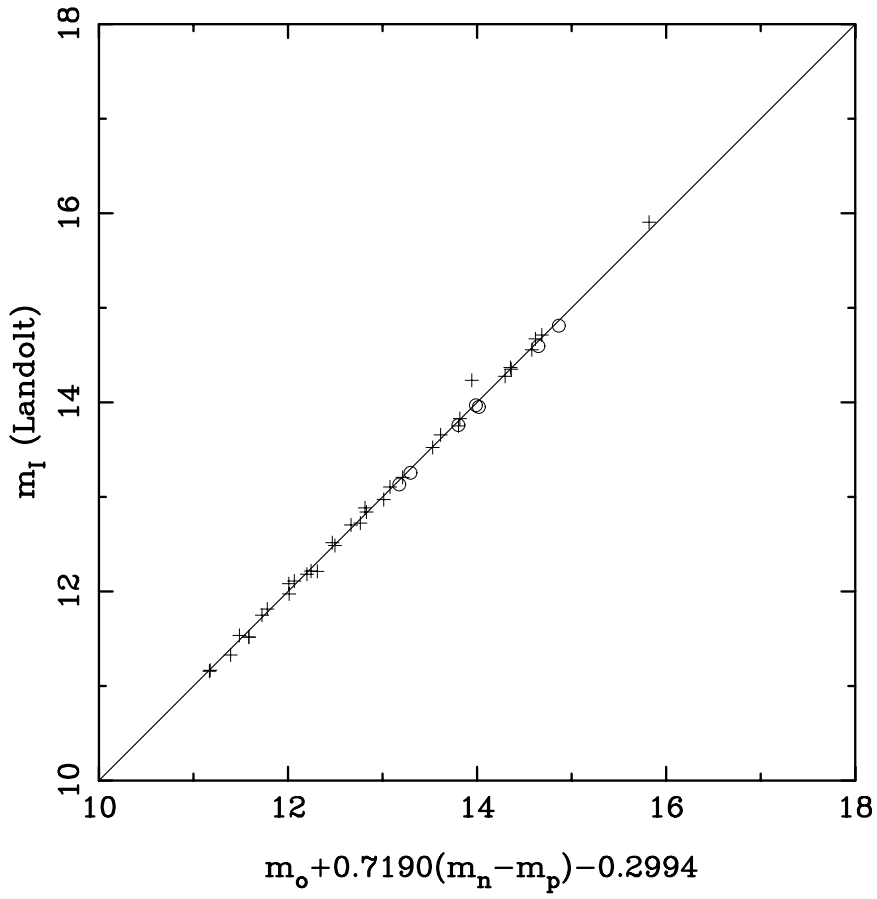

Fig. 11. The comparison between Landolt $I$ magnitude and the BATC $o, n$ and $p$ magnitudes. Here $o(918 \mathrm{~nm})$ band is nearest to $I$ band, and $n(848 \mathrm{~nm})$ and $p(974 \mathrm{~nm})$ bands are used for colour correction.

Table 3. The fitting statistics of the $U B V R I$ from BATC photometry. Column 1 lists the name of the band. Column 2 lists the number of the stars used for fitting. Columns 3 and 4 list the ranges of color index of each band, and the last column lists the rms fitting errors.

\begin{tabular}{ccccc}
\hline \hline filter & No. of stars & $(B-V)_{\min }$ & $(B-V)_{\max }$ & Error \\
\hline$U$ & 45 & -0.22 & 2.00 & 0.143 \\
$B$ & 37 & -0.22 & 1.76 & 0.076 \\
$V$ & 33 & -0.22 & 1.53 & 0.027 \\
$R$ & 26 & -0.22 & 1.53 & 0.055 \\
$I$ & 40 & -0.22 & 1.76 & 0.064 \\
\hline
\end{tabular}

to measure the magnitude of faint objects whose PSFs were difficult to get. By doing so, we have obtained the magnitude estimates for the majority of the stars in the field and the number of objects missed by both methods should be insignificant.

\section{System conversion}

In our previous work (Fan et al. 1996), we made a comparison of magnitudes between the BATC system and the UBVRI system through the stars in the field of open star cluster M67 (Gilliland 1991).

In the papers of Landolt $(1983,1992)$ and of GaladíEnríquez et al. (2000), there exists $U B V R I$ photometry for 55 stars in the field of Landolt SA95. By cross-checking the published catalogues of the standards and our SED catalogue, we found 48 stars in common, since the other 7 stars are too bright and saturated in our images in most bands. In Tables 4 and 5, there are 42 stars taken from SA95 field of
Table 4. The positions and names of the SA95 standard stars used in system conversion. Column 1 is the sequence number in increasing right arsension $(\alpha)$. Columns 2 and 3 are the equatorial coordinates in epoch 2000.0. Last 3 columns give the names in catalogues of Landolt (1983), Landolt (1992) and Galadí-Enríquez et al. (2000).

\begin{tabular}{|c|c|c|c|c|c|}
\hline $\begin{array}{l}\text { No. } \\
\text { (1) }\end{array}$ & $\begin{array}{c}\alpha(2000.0) \\
(2)\end{array}$ & $\begin{array}{c}\delta(2000.0) \\
(3)\end{array}$ & $\begin{array}{l}\text { L1992 } \\
\text { (4) }\end{array}$ & $\begin{array}{c}\text { G2000 } \\
\text { (5) }\end{array}$ & $\begin{array}{l}\text { L1983 } \\
(6)\end{array}$ \\
\hline 01 & $03: 52: 34.01$ & $-00: 02: 27.3$ & & $3-1$ & \\
\hline 02 & 03:52:36.96 & $-00: 03: 32.5$ & & $3-2$ & \\
\hline 03 & 03:52:39.95 & $-00: 03: 07.8$ & & $3-3$ & \\
\hline 04 & 03:52:40.31 & $-00: 05: 23.3$ & $95-015$ & & \\
\hline 05 & 03:52:40.62 & $-00: 05: 06.1$ & $95-016$ & & \\
\hline 06 & 03:52:41.17 & $00: 31: 20.7$ & $95-301$ & & SA95-301 \\
\hline 07 & $03: 52: 42.17$ & $00: 31: 17.1$ & $95-302$ & & \\
\hline 08 & $03: 52: 44.82$ & $-00: 03: 34.1$ & & $3-4$ & \\
\hline 09 & 03:52:53.10 & $-00: 03: 52.4$ & & $3-5$ & \\
\hline 10 & $03: 52: 54.18$ & 00:00:18.4 & $95-096$ & & SA95-096 \\
\hline 11 & 03:52:54.87 & $-00: 03: 16.1$ & & $3-6$ & \\
\hline 12 & 03:52:57.50 & $-00: 00: 19.8$ & $95-097$ & $3-7$ & \\
\hline 13 & 03:53:00.23 & 00:02:48.5 & $95-098$ & & \\
\hline 14 & 03:53:00.78 & 00:00:15.2 & $95-100$ & & \\
\hline 15 & 03:53:04.14 & 00:02:49.5 & $95-101$ & & \\
\hline 16 & 03:53:07.58 & 00:01:10.3 & $95-102$ & & \\
\hline 17 & 03:53:10.62 & $00: 27: 22.3$ & $95-252$ & & \\
\hline 18 & 03:53:13.24 & $00: 16: 22.2$ & $95-190$ & & \\
\hline 19 & 03:53:20.59 & $00: 16: 34.3$ & $95-193$ & & \\
\hline 20 & $03: 53: 21.32$ & $-00: 00: 19.0$ & $95-105$ & & \\
\hline 21 & $03: 53: 25.18$ & 00:01:22.2 & $95-106$ & & \\
\hline 22 & $03: 53: 25.65$ & 00:02:19.8 & $95-107$ & & \\
\hline 23 & 03:53:40.10 & $-00: 01: 11.9$ & $95-112$ & & \\
\hline 24 & 03:53:41.19 & $-00: 02: 32.8$ & $95-041$ & & \\
\hline 25 & 03:53:43.66 & $-00: 04: 33.9$ & $95-042$ & & \\
\hline 26 & 03:53:44.17 & $00: 29: 49.5$ & $95-317$ & & \\
\hline 27 & 03:53:46.98 & $00: 26: 40.4$ & $95-263$ & & \\
\hline 28 & 03:53:47.80 & $-00: 00: 47.8$ & $95-115$ & & \\
\hline 29 & 03:53:48.59 & $-00: 03: 02.0$ & $95-043$ & & \\
\hline 30 & 03:54:16.28 & 00:18:51.8 & $95-271$ & & \\
\hline 31 & 03:54:19.45 & $00: 36: 31.3$ & $95-328$ & & \\
\hline 32 & 03:54:23.89 & $00: 37: 06.7$ & $95-329$ & & \\
\hline 33 & $03: 54: 30.73$ & $00: 29: 03.5$ & $95-330$ & & \\
\hline 34 & $03: 54: 44.23$ & $00: 27: 19.2$ & $95-275$ & & \\
\hline 35 & 03:54:45.86 & $00: 25: 53.3$ & $95-276$ & & \\
\hline 36 & $03: 54: 49.52$ & $-00: 07: 04.4$ & $95-060$ & & \\
\hline 37 & 03:54:49.93 & 00:10:08.0 & $95-218$ & & \\
\hline 38 & 03:54:51.66 & 00:05:21.1 & $95-132$ & & SA95-132 \\
\hline 39 & 03:55:00.39 & $-00: 02: 54.5$ & $95-62$ & & \\
\hline 40 & 03:55:03.72 & 00:03:26.4 & $95-137$ & & \\
\hline 41 & 03:55:04.65 & 00:03:07.6 & $95-139$ & & \\
\hline 42 & 03:55:08.73 & $00: 14: 34.0$ & $95-227$ & & \\
\hline 43 & 03:55:09.37 & 00:01:20.2 & $95-142$ & & \\
\hline 44 & 03:55:31.14 & $-00: 09: 13.8$ & $95-074$ & & SA95-074 \\
\hline 45 & 03:55:38.81 & $00: 10: 43.0$ & $95-231$ & & \\
\hline 46 & $03: 55: 41.54$ & $00: 26: 36.9$ & $95-284$ & & \\
\hline 47 & 03:55:44.10 & $00: 25: 09.6$ & $95-285$ & & \\
\hline 48 & $03: 55: 44.42$ & 00:07:02.3 & $95-149$ & & \\
\hline
\end{tabular}


Table 5. The SED of the Landolt standard stars. The value of 00.00 means that there is no measurement because the star is saturated in this band. The magnitude error of such star is estimated to be 0.03 .

\begin{tabular}{|c|c|c|c|c|c|c|c|c|c|c|c|c|c|c|c|}
\hline $\begin{array}{l}\text { N. } \\
\text { (1) }\end{array}$ & $\begin{array}{c}a \\
\text { (2) }\end{array}$ & $\begin{array}{c}b \\
\text { (3) }\end{array}$ & $\begin{array}{c}c \\
\text { (4) }\end{array}$ & $\begin{array}{c}d \\
(5)\end{array}$ & $\begin{array}{c}e \\
(6)\end{array}$ & $\begin{array}{c}f \\
\text { (7) }\end{array}$ & $\begin{array}{c}g \\
(8)\end{array}$ & $\begin{array}{c}h \\
(9)\end{array}$ & $\begin{array}{c}i \\
(10)\end{array}$ & $\begin{array}{c}j \\
(11)\end{array}$ & $\begin{array}{c}k \\
(12)\end{array}$ & $\begin{array}{c}m \\
(13)\end{array}$ & $\begin{array}{c}n \\
(14)\end{array}$ & $\begin{array}{c}o \\
(15)\end{array}$ & $\begin{array}{c}p \\
(16)\end{array}$ \\
\hline 01 & 17.39 & 16.54 & 16.24 & 15.86 & 15.66 & 15.52 & 15.29 & 15.23 & 15.12 & 15.06 & 15.04 & 14.95 & 14.95 & 14.89 & 14.88 \\
\hline 02 & 17.45 & 16.66 & 16.07 & 15.50 & 15.23 & 15.05 & 14.74 & .69 & 14.55 & .49 & 1 & 33 & 31 & 14.23 & .24 \\
\hline 03 & 15.85 & 15.08 & 14.77 & 14.44 & 14.21 & 14.12 & 13.91 & 88 & 13.79 & 13.74 & 13.73 & 13.61 & 13.62 & 13.56 & 13.58 \\
\hline 04 & 13.30 & 12.57 & 12.13 & 11.71 & 00.00 & 00.00 & 00.00 & 00.00 & 00.00 & 00.00 & 00.00 & 00.00 & 00.00 & 10.81 & 10.83 \\
\hline 05 & 17.92 & 16.66 & 16.03 & 15.05 & 14.72 & 14.56 & 13.97 & 13.82 & 13.58 & 13.48 & 13.34 & 13.16 & 13.13 & 13.00 & 12.96 \\
\hline 06 & 15.23 & 13.85 & 13.06 & 12.15 & 00.00 & 11.46 & 11.09 & 00.00 & 00.00 & 00.00 & 00.00 & 00.00 & 00.00 & 00.00 & 10.00 \\
\hline 07 & 14.20 & 13.33 & 12.83 & 12.21 & 00.00 & 00.00 & 00.00 & 00.00 & 00.00 & 00.00 & 00.00 & 00.00 & 00.00 & 11.08 & 11.08 \\
\hline 08 & 18.42 & 17.61 & 16.99 & 16.41 & 16.07 & 15.96 & 15.65 & 15.57 & 15.41 & 15.35 & 15.29 & 15.18 & 15.17 & 15.07 & 15.05 \\
\hline 09 & 16.61 & 15.72 & 15.43 & 15.17 & 14.94 & 14.85 & 14.64 & 14.60 & 14.50 & 14.42 & 14.41 & 14.32 & 14.33 & 14.26 & 14.26 \\
\hline 10 & 11.49 & 10.22 & 10.25 & 10.36 & 00.00 & 00.00 & 00.00 & 00.00 & 00.00 & 00.00 & 00.00 & 00.00 & 00.00 & 10.17 & 10.21 \\
\hline 11 & 16.15 & 15.35 & 14.89 & 14.53 & 14.22 & 14.13 & 13.88 & 13.83 & 13.70 & 13.62 & 13.58 & 13.48 & 13.49 & 13.41 & 13.41 \\
\hline 12 & 17.25 & 16.31 & 15.86 & 15.40 & 15.10 & 14.96 & 14.65 & 14.58 & 14.42 & 14.35 & 14.29 & 14.15 & 14.14 & 14.01 & 14.02 \\
\hline 13 & 17.68 & 16.93 & 16.03 & 15.17 & 14.84 & 14.72 & 14.18 & 14.05 & 13.85 & 13.75 & 13.63 & 13.49 & 13.48 & 13.27 & 13.34 \\
\hline 14 & 17.66 & 16.90 & 16.49 & 16.10 & 15.79 & 15.70 & 15.44 & 15.37 & 15.23 & 15.18 & 15.11 & 14.99 & 14.98 & 14.86 & 14.91 \\
\hline 15 & 14.79 & 14.09 & 13.57 & 13.14 & 12.89 & 12.77 & 12.52 & 00.00 & 00.00 & 00.00 & 00.00 & 12.17 & 12.18 & 12.03 & 12.12 \\
\hline 16 & 17.87 & 17.18 & 16.68 & 16.23 & 15.93 & 15.78 & 15.48 & 15.40 & 15.24 & 15.18 & 15.12 & 14.98 & 14.95 & 14.80 & 14.85 \\
\hline 17 & 19.59 & 18.17 & 17.38 & 16.44 & 15.91 & 15.67 & 15.13 & 14.94 & 14.67 & 14.52 & 14.32 & 14.20 & 14.11 & 13.93 & 13.86 \\
\hline 18 & 14.46 & 13.02 & 12.91 & 12.74 & 00.00 & 00.00 & 00.00 & 00.00 & 00.00 & 00.00 & 00.00 & 12.57 & 12.60 & 12.51 & 12.47 \\
\hline 19 & 17.83 & 16.91 & 16.05 & 15.09 & 14.71 & 14.61 & 14.04 & 13.89 & 00.00 & 13.58 & 13.43 & 13.33 & 13.28 & 13.19 & 13.12 \\
\hline 20 & 16.31 & 15.56 & 14.82 & 14.18 & 13.85 & 13.74 & 13.38 & 13.29 & 00.00 & 13.05 & 12.97 & 12.88 & 12.86 & 12.71 & 12.75 \\
\hline 21 & 18.06 & 17.13 & 16.49 & 15.90 & 15.56 & 15.35 & 15.00 & 14.88 & 14.68 & 14.61 & 14.53 & 14.39 & 14.33 & 14.15 & 14.21 \\
\hline 22 & 19.84 & 19.02 & 18.29 & 17.28 & 16.91 & 16.65 & 16.02 & & 15.50 & 29 & 94 & 85 & .75 & 14.43 & .45 \\
\hline 23 & 17.82 & 17.01 & 16.61 & 16.12 & 15.77 & 15.60 & 15.29 & 15.21 & 15.00 & 14.91 & 14.81 & 14.70 & 14.65 & 14.51 & 14.54 \\
\hline 24 & 16.50 & 15.66 & 15.16 & 14.70 & 14.35 & 14.18 & 13.86 & 13.76 & 13.56 & 13.46 & 13.36 & 13.25 & 13.19 & 13.02 & 13.07 \\
\hline 25 & 15.09 & 15.14 & 15.29 & 15.35 & 15.39 & 15.51 & 15.70 & 15.79 & 15.98 & 16.02 & 16.16 & 16.26 & 16.39 & 16.32 & 16.68 \\
\hline 26 & 17.23 & 16.03 & 15.22 & 14.41 & 14.02 & 13.69 & 13.19 & 13.01 & 00.00 & 12.66 & 00.00 & 12.34 & 12.25 & 12.16 & 12.05 \\
\hline 27 & 17.18 & 15.73 & 14.72 & 13.75 & 13.19 & 12.95 & 12.39 & 12.20 & 11.93 & 00.00 & 00.00 & 00.00 & 11.43 & 11.29 & 11.18 \\
\hline 28 & 17.18 & 16.37 & 15.85 & 15.36 & 15.01 & 14.82 & 14.50 & 14.42 & 14.21 & 14.13 & 14.02 & 13.91 & 13.84 & 13.75 & 13.74 \\
\hline 29 & 12.37 & 11.61 & 11.33 & 11.25 & 00.00 & 00.00 & 00.00 & 00.00 & 00.00 & 00.00 & 00.00 & 00.00 & 00.00 & 10.49 & 10.57 \\
\hline 30 & 17.07 & 16.02 & 15.24 & 14.59 & 14.14 & 13.87 & 13.44 & 13.30 & 00.00 & 00.00 & 12.77 & 12.59 & 12.50 & 12.37 & 12.27 \\
\hline 31 & 17.70 & 16.39 & 15.53 & 14.67 & 14.14 & 13.78 & 13.22 & 13.02 & 12.66 & 00.00 & 00.00 & 12.08 & 11.98 & 11.78 & 11.65 \\
\hline 32 & 17.98 & 17.17 & 16.28 & 15.37 & 15.06 & 14.89 & 14.32 & 14.15 & 13.91 & 13.83 & 13.65 & 13.52 & 13.49 & 13.36 & 13.29 \\
\hline 33 & 17.62 & 16.28 & 14.94 & 13.72 & 00.00 & 12.56 & 00.00 & 00.00 & 00.00 & 00.00 & 00.00 & 00.00 & 00.00 & 00.00 & 09.66 \\
\hline 34 & 18.33 & 16.88 & 15.78 & 14.78 & 14.18 & 13.79 & 13.10 & 00.00 & 00.00 & 00.00 & 00.00 & 00.00 & 11.72 & 11.52 & 11.36 \\
\hline 35 & 17.69 & 16.73 & 15.79 & 14.90 & 14.54 & 14.40 & 13.84 & 13.68 & 00.00 & 13.37 & 13.19 & 13.06 & 13.02 & 12.92 & 12.83 \\
\hline 36 & 15.48 & 14.83 & 14.38 & 13.91 & 13.55 & 13.52 & 13.26 & 13.20 & 00.00 & 13.00 & 12.92 & 12.87 & 12.83 & 12.76 & 12.83 \\
\hline 37 & 14.11 & 13.35 & 12.88 & 12.51 & 00.00 & 00.00 & 00.00 & 00.00 & 00.00 & 00.00 & 00.00 & 00.00 & 11.68 & 11.65 & 11.63 \\
\hline 38 & 14.09 & 12.79 & 12.51 & 12.29 & 00.00 & 00.00 & 00.00 & 00.00 & 00.00 & 00.00 & 00.00 & 00.00 & 11.90 & 11.82 & 11.82 \\
\hline 39 & 17.37 & 16.26 & 15.41 & 14.50 & 14.00 & 13.76 & 13.27 & 13.15 & 12.85 & 00.00 & 12.61 & 12.49 & 12.40 & 12.23 & 12.22 \\
\hline 40 & 18.17 & 17.20 & 16.50 & 15.45 & 15.06 & 14.82 & 14.17 & 13.99 & 13.69 & 13.53 & 13.25 & 13.11 & 13.01 & 12.83 & 12.83 \\
\hline 41 & 14.88 & 14.17 & 13.47 & 12.75 & 00.00 & 00.00 & 00.00 & 00.00 & 00.00 & 00.00 & 00.00 & 00.00 & 11.52 & 11.41 & 11.45 \\
\hline 42 & 17.65 & 16.86 & 16.63 & 16.32 & 16.05 & 15.88 & 15.63 & 15.53 & 15.36 & 15.28 & 15.19 & 15.07 & 15.01 & 14.89 & 14.89 \\
\hline 43 & 14.75 & 13.85 & 13.63 & 13.29 & 13.06 & 13.00 & 12.81 & 00.00 & 00.00 & 00.00 & 12.68 & 12.57 & 12.54 & 12.47 & 12.51 \\
\hline 44 & 14.51 & 13.61 & 13.10 & 12.32 & 00.00 & 00.00 & 00.00 & 00.00 & 00.00 & 00.00 & 00.00 & 00.00 & 00.00 & 10.59 & 10.56 \\
\hline 45 & 16.18 & 14.96 & 14.75 & 14.46 & 14.29 & 14.25 & 14.14 & 14.11 & 14.10 & 14.04 & 14.03 & 14.00 & 13.99 & 13.87 & 13.94 \\
\hline 46 & 17.45 & 16.35 & 15.53 & 14.69 & 14.19 & 13.92 & 13.38 & 13.22 & 00.00 & 00.00 & 00.00 & 12.41 & 12.35 & 12.10 & 12.07 \\
\hline 47 & 17.96 & 16.95 & 16.65 & 16.22 & 15.86 & 15.68 & 15.35 & 15.24 & 15.05 & 14.98 & 14.81 & 14.68 & 14.66 & 14.51 & 14.46 \\
\hline 48 & 15.44 & 14.09 & 13.13 & 12.12 & 00.00 & 00.00 & 00.00 & 00.00 & 00.00 & 00.00 & 00.00 & 00.00 & 00.00 & 00.00 & 00.00 \\
\hline
\end{tabular}


Landolt (1992) which includes 4 stars of Landolt (1992) and all 7 stars in 3rd field of Galadi-Enriquez et al. (2000).

The coordinates of one of the stars, 95-285, may be wrong. At $\alpha=03 \mathrm{~h} 55 \mathrm{~m} 46 \mathrm{~s}, \delta=+00^{\circ} 23^{\prime} 40^{\prime \prime}$ (2000.0), we did not find any object. From the finding chart of Landolt (1992) and the given magnitude, we suggest that the object 95-285 should be located at $03 \mathrm{~h} 55 \mathrm{~m} 44.1,+00^{\circ} 25^{\prime} 09^{\prime} .^{\prime} 6$ (2000.0).

By comparison of the $U B V R I$ and the BATC magnitudes of these 48 stars, we obtained the relationships between the two photometric systems via a linear fitting method, presented in Figs. 7-11, respectively.

$m_{U}=m_{b}+0.6801\left(m_{a}-m_{b}\right)-0.8982 \pm 0.143$,
$m_{B}=m_{d}+0.2201\left(m_{c}-m_{e}\right)+0.1278 \pm 0.076$,
$m_{V}=m_{g}+0.3292\left(m_{f}-m_{h}\right)+0.0476 \pm 0.027$,
$m_{R}=m_{i}+0.1036 \pm 0.055$,
$m_{I}=m_{o}+0.7190\left(m_{n}-m_{p}\right)-0.2994 \pm 0.064$.

In Figs. 7-11 we use different symbols for the points from the catalogues of Landolt $(1983,1992)$ and from the catalogue of Galadí-Enríquez et al. (2000). By careful inspection of the figures, we did not find any systematic difference.

Some of the standards are too bright and are out of our magnitude ranges. We can thus only use part of the Landolt standards to perform fitting. Table 3 list useful information for the fitting process, which includes the number of stars used, the range of colour index $B-V$ and the errors of the fitting in each band of the $U B V R I$ system.

The comparison and fitting between $V$ band and the BATC $g, f$ and $h$ bands presents the best case in this study, and the rms is about 0.027 mag. The RMSs of fitting for the other broadbands are somewhat larger.

To obtain transformation equations for the derivation of approximate $U B V R I$ magnitudes and colours from BATC photometry is important as well as essential. The above relationships can be used for the system transformation and can also be applied to the verification of our internal estimation of accuracy by using Landolt's catalogues. Although the many comparisons and fits between the two systems are good and self-consistent, the study will not be limited to this single field. The BATC survey program has been and will be observing more target fields including other standard fields. Additional comparisons in the future with more stars over larger colour range will provide better information as to how the magnitude measurements can be transformed between various observing systems.

\section{Conclusion}

On a total of 41 nights in the period from December 13, 1994 to December 16, 1999, we made observations of the Landolt SA95 field. A total of 189 images with acceptable quality were selected for photometric measurements. A complete SED catalogue in 15 colours in the BATC system for the stars in the field of Landolt SA95 is presented. The wavelength coverage with the 15 intermediate filter bands from is $300 \mathrm{~nm}$ to $1000 \mathrm{~nm}$. The visual magnitude ranges from 10th to 20th mag. We describe the methods of observation and data reduction, and analysed the possible error of our measurement. By comparison with Landolts $U B V R I$ broad band photometric magnitudes of 48 stars, the relationships between the BATC intermediateband system and Landolt $U B V R I$ broad band photometic system are obtained. A catalogue has been created in which a total of 3613 stars are included. The catalogue is also published in electronic form and is available at the CDS ftp site: cdsarc.u-strasbg.fr (130.79.128.5).

Acknowledgements. We are indebted to the anonymous referee for many critical comments and helpful suggestions, and for English editing that has greatly improved our paper. We thank Yao-Hua Li for his management and support of the instruments. We also thank the assistants who helped with the observations for their hard work and kind cooperation. Wei-Hsin Sun acknowledges the support from National Science Council under the grant NSC 89-2112-M-008-021.

\section{References}

Castelli, F., \& Kurucz, R. L. 1994, A\&A, 281, 817

Fan, X., Burstein, D., Chen, J., et al. 1996, AJ, 112, 628

Fukugita, M., Ichikawa, T., Gunn, J. E., et al. 1996, AJ, 111, 1748

Galadí-Enríquez, D., Trullols, E., \& Jordi, C. 2000, A\&AS, 146, 169

Gilliland, R. L., Brown, T. M., Duncan, D. K., et al. 1991, AJ, 101, 541

Jenkner, H., Lasker, B. M., Sturch, C. R., et al. 1990, AJ, 99, 2082

Landolt, A. U. 1983, AJ, 88, 439

Landolt, A. U. 1992, AJ, 104, 340

Oke, J. B., \& Gunn, J. E. 1983, ApJ, 266, 713

Oke, J. B. 1990, AJ, 99, 1621

Stetson, P. B. 1987, PASP, 99, 191

Yan, H., Burstein, D., Fan, X., et al. 2000, PASP, 112, 691

Zhou, X., Chen, J., Xu, W., et al. 1999, PASP, 111, 909

Zhou, X., Jiang, Z., Xue, S., et al. 2001, CJAA, 1, 372

Zhou, X., et al. 2002, in preparation 